\title{
Multidermatomal nevus comedonicus: How dermoscopy aids diagnosis?
}

\section{Guneet Awal, Tanreet Kaur}

\author{
Department of Dermatology, Sri Guru Ram Das Institute of Medical Sciences \& Research, Sri Amritsar, Punjab, India
}

Corresponding author: Dr. Guneet Awal, E-mail: guneetawal@gmail.com

\begin{abstract}
Nevus Comedonicus (NC)is a rare subtype of epidermal nevus considered as a variant of adnexal hamartoma that presents as grouped hyperkeratotic papular lesions with appearance similar to comedones. Lesions usually present at birth but involvement can occur any time up to middle age. Here we report a case of NC in 16 year old female occurring after puberty. We report this case because of its multidermatomal involvement, strictly unilaterally distributed lesions with progressive pubertal enlargement showing characteristic features of $\mathrm{NC}$ on dermoscopy, thereby helping to differentiate it from other diseases with similar clinical presentation.
\end{abstract}

Key words: Epidermal nevus; Nevus comedonicus; Comedo like

\section{INTRODUCTION}

$\mathrm{NC}$ is uncommon anomaly affecting pilosebaceous unit in which follicular structure instead of forming hair shaft produces keratin plug occluding the follicle which presents as linear or grouped hyperpigmented comedo like papular lesions $[1,2]$. It most commonly involves face, head and neck region [3]. Incidence is equal in males and females with most of the cases occurring before 10 years of age [4]. Lesions can get secondarily infected or may develop pilar sheath acanthomas and trichofolliculomas [5]. In current scenario, dermoscopy being non-invasive and simple diagnostic tool plays pivotal role in diagnosing and ruling out clinically similar differentials.

\section{CASE REPORT}

A 16 year old female presented in the dermatology outpatient with asymptomatic multiple grouped hyperpigmented keratotic follicular lesions since the age of 11 years distributed strictly unilaterally over right side involving chest, mammary area, axilla, right arm spreading to involve back with multidermatomal distribution (cervical C4,C5 and thoracic T1-T7) (Fig. 1). Lesions were completely asymptomatic not associated with any complaint of itching, oozing or ulceration. Only concern of the patient was cosmetic appearance as these lesions progressively enlarged during last 3-4 years. No history of any lesions at time of birth, precipitating trauma or rash given by the patient. There was no similar family history and patient was born of non-consanguineous marriage. No other associated ocular, CNS or skeletal abnormalities were observed. All routine investigations were within normal limits. Lesions were examined using DermLite DL3 Dermoscope which revealed multiple dark brown areas studded with keratin plugs and numerous follicular openings showing characteristic dark colored plugging (Fig. 2). These changes were consistent with the clinical diagnosis of Nevus Comedonicus, in contrast to acne vulgaris where superficial circular areas of homogeneous hyperpigmention are usually seen. Skin biopsy done from the lesions present over the upper back revealed large dilated poorly formed follicles filled with lamellated orthokeratotic material (Fig. 3).

\section{DISCUSSION}

Nevus Comedonicus, also known as Zoniform Nevus is considered as adenexal hemartoma with developmental

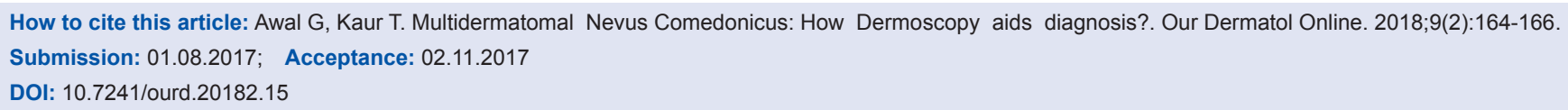




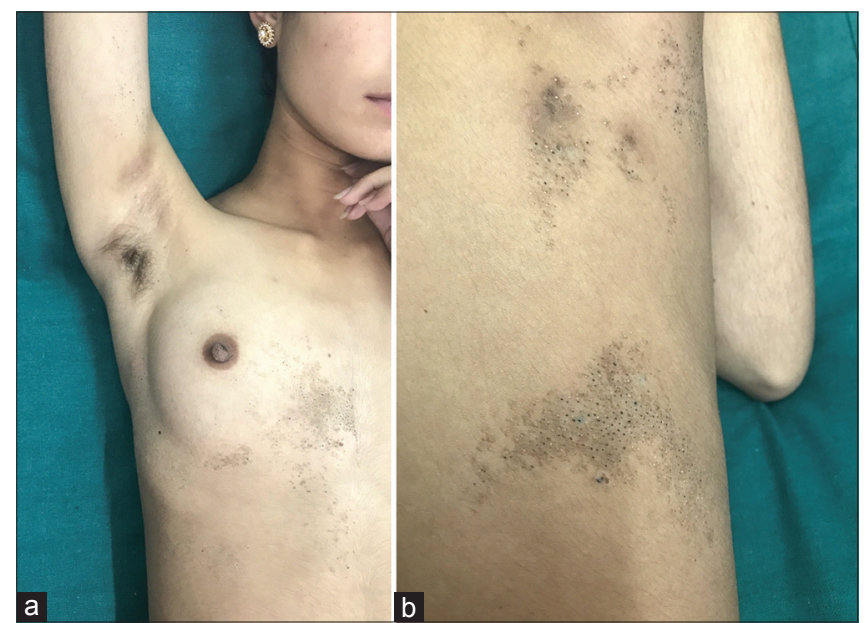

Figure 1: (a) Hyperkeratotic grouped comedo like papular lesions distributed unilaterally over right side involving chest, axilla and right arm. (b) Back of the patient showing keratotic plugging distributed unilaterally in a grouped pattern.

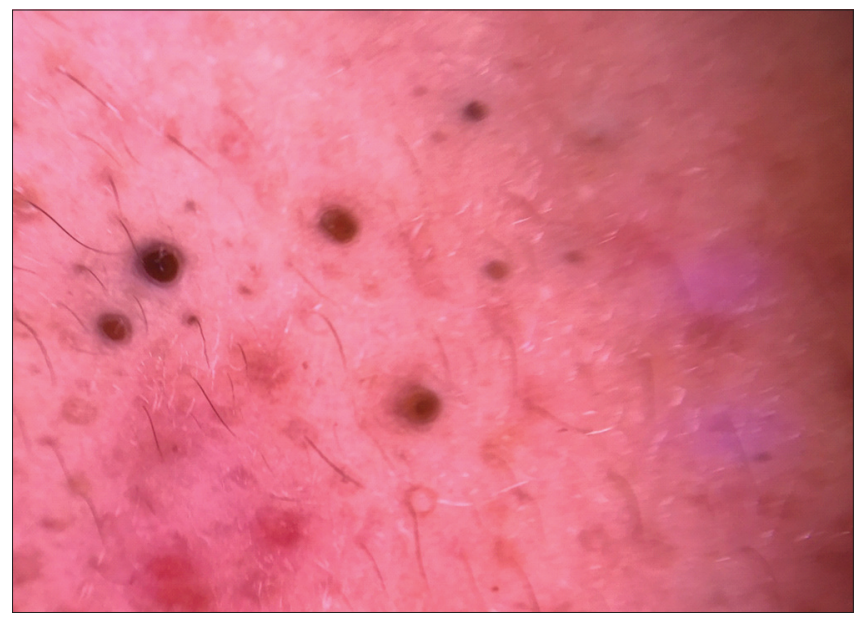

Figure 2: Dermoscopic picture of NC done from back of the patient showing multiple dark brown colored keratotic plugs.

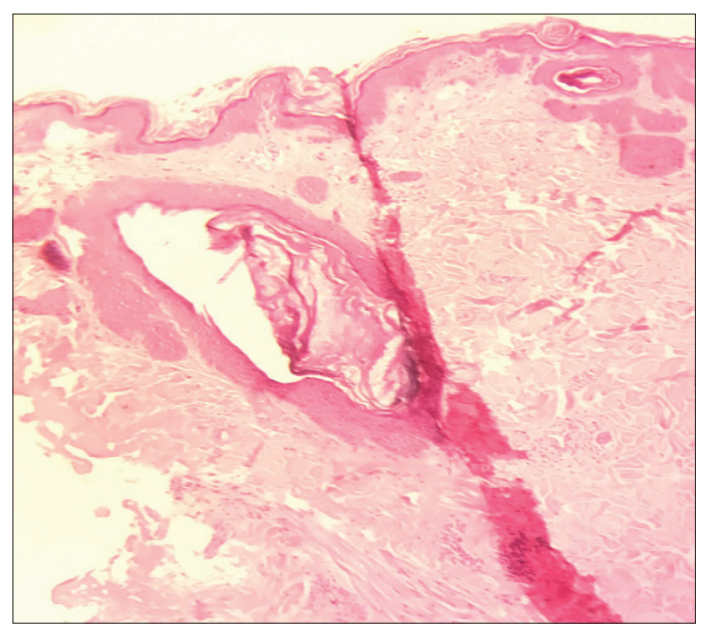

Figure 3: Histopathology of NC showing hair follicle filled with lamellated keratotic material with normal interfollicular epidermis. failure of pilosebaceous unit. It is a misnomer as true comedones are absent [6]. Two types of NC have been reported [7]. First type is characterised by asymptomatic comedo like lesions distributed in various patterns like linear, interrupted, unilateral or bilateral which mainly is of cosmetic concern to the patients, where as second type is characterised by cysts, abscesses, fistulas and scarring mainly because of recurrent secondary infections and inflammation. The Case reported here is consistent with the presentation of first type with unilateral multidermatomal involvement.

Dermoscopy is a non invasive and confirmatory tool to rule out differentials of NC. It is recordable, repeatable and bed side investigation. Till now very few reports have been published in context of dermoscopic differentials of $\mathrm{NC}$ [8]. In acne, dermoscopic features depend upon comedone type whether open or closed. In contrast to $\mathrm{NC}$, where follicular openings studded with keratin plugs are characteristic, in acne vulgaris circular homogeneous more superficially located areas of hyperpigmentation are seen. Other differentials includes sebaceous nevus where aggregated bright yellow dots without any hair follicles are seen. Rarely hair follicle nevus showing pseudo-pigmented network is seen on dermoscopy. In this case dermoscopic features characterically revealed keratin plugs consistent with NC.

Histopathologically lesions of NC typically reveal dilated hair follicles containing keratinous debris with acanthotic or hyperkeratotic interfollicular epidermis. In the current case inter-follicular epidermis is normal.

$\mathrm{NC}$ can be a part of epidermal nevus syndrome which is associated with several ocular abnormalities which includes cataract, corneal involvement, skeletal anomalies like hemivertebrae, absence of little finger, scoliosis and various neurological abnormalities. In long standing cases of NC follicular tumors have been reported [9]. Our patient did not have any such association thus it is not a part of nevus comedonicus syndrome.

Treatment is mainly aimed for cosmetic purposes. In this patient we tried $0.1 \%$ tretinoin cream for 4 weeks and two sittings with Fractional C02 laser were done, after which the patient was lost to follow up. Other topical modalities that can be used include adaplalene, tazarotene, calcipotriene alone or in combination with steroids [10]. In resistant cases mechanical removal of plugs using cosmetic strips or dermabrasion can 
be tried. Various lasers such as Er-YAG laser, diode laser, CO2 laser have been therapeutically explored for treating $\mathrm{NC}$ with varied results [11]. Use of oral isotretinoin has been tried for treating few cases of extensive NC. Surgical excision has been kept as last resort or in cases of localised tumor growth or inflammation.

\section{REFERENCES}

1. Beerman HB, Van Meter RH. Nevus comedonicus. Arch Derm Syphilol. 1949;60:130.

2. Sharma RP, Singh SP. Extensive unilateral nevus comedonicus with bilateral involvement of face. Indian J Dermatol Venereol Leprol. 2001;67:195-6.

3. Vasiloudes PE, Morelli JG, Weston WL. Inflammatory nevus comedonicus in children. J Am Acad Dermatol. 1998;38:834-6.

4. Givan J, Hurley MY, Glaser DA. Nevus comedonicus: a novel approach to treatment. Dermatol Surg. 2010;36:721-5.

5. Dudley K, Barr WG, Amrin A, Massa MC. Nevus comedonicus in association with widespread, well differentiated follicular tumors. J Am Acad Dermatol. 1986;15:1123-7.

6. Lefkowitz A, Schwartz RA, Lambert WC. Nevus comedonicus. Dermatology. 1991;199:204-7.

7. Inoue Y, Miyamoto Y, Ono Y. Two cases of Nevus Comedonicus: Successful treatment of keratin plugs with a pore strip. J Am Acad Dermatol. 2000;43:927-9.

8. Vano -Galvan S, Hernandez-Martin A. Disseminated congenital comedones. Pediatr Dermatol. 2011;28:58-9.

9. Sugarman JL. Epidermal nevus syndromes. Semin Cutan Med Surg. 2007;26:221-30.

10. Milton GP, DiGiovanna JJ, Peck GL. Treatment of nevus comedonicus with ammonium lactate lotion. J Am Acad Dermatol. 1989;20:324-8.

11. Milburn S, Whallett E, Hancock K, Munnoch DA, Stevenson JH. The treatment of nevus comedonicus. Br J Plast Surg. 2004;57:805-6.

Copyright by Guneet Awal, et al. This is an open-access article distributed under the terms of the Creative Commons Attribution License, which permits unrestricted use, distribution, and reproduction in any medium, provided the original author and source are credited.

Source of Support: Nil, Conflict of Interest: None declared. 